\title{
Atomic screening of nuclear transitions
}

\author{
B. R. Davis and S. E. Koonin \\ W. K. Kellogg Radiation Laboratory, California Institute of Technology, Pasadena, California 91125 \\ P. Vogel \\ Department of Physics, California Institute of Technology, Pasadena, California 91125 \\ (Received 12 May 1980)
}

\begin{abstract}
In the analysis of time-reversal and Mössbauer absorption experiments, it is important to consider atomic processes which interfere with the direct nuclear transition. Interaction of the photon with the atomic electrons causes the radiation to acquire a phase shift, specified by the interference parameter $\xi(L \pi)$. We present theoretical expressions for $\xi$ and compare our calculated values with experiment. Satisfactory agreement is obtained. In particular, an apparent violation of time-reversal invariance in the $129-\mathrm{keV}$ transition of ${ }^{191} \mathrm{Ir}$ is fully explained by these effects.
\end{abstract}

$\left[\begin{array}{c}\text { RADIOACTIVITY Atomic effects on nuclear electromagnetic transitions; time- } \\ \text { reversal violation; dispersion of Mössbauer lines. }\end{array}\right]$

\section{INTRODUCTION}

Photons emitted or absorbed in a nuclear transition may interact with the atomic electrons. Such processes will interfere with direct photon emission or absorption if the final states are indistinguishable from each other. In this paper, we consider processes which shift the photon phase by an amount of the order of the fine structure constant. This phase shift, $\xi(L, \pi=E$ or $M)$, depends upon both the multipolarity $(L \pi)$ and the energy of the nuclear transition, and has been called the "screening" or "interference" parameter. ${ }^{1-4}$

There are two types of experiments for which a knowledge of $\xi$ is important. The first of these tests time-reversal invariance in a mixed-multipole $\left(L \pi\right.$ and $\left.L^{\prime} \pi^{\prime}\right)$ electromagnetic decay of an excited nucleus. One seeks to measure a relative phase between the transition matrix elements of the two competing multipoles. This phase, $\eta$ $\equiv \eta(L \pi)-\eta\left(L^{\prime} \pi^{\prime}\right)$, makes the mixing ratio $\delta$ complex:

$$
\delta= \pm|\delta| e^{i \eta} .
$$

A nonvanishing value of $\eta$ would be evidence for time-reversal violation. ${ }^{5}$ However, the mixing ratio can acquire a spurious phase resembling $\eta$ through the interaction of the radiated photon with the surrounding atomic electrons. This process, also sometimes called the "final state interaction," is shown schematically in Fig. 1. Diagram (a) represents the amplitude for direct emission of a photon of multipolarity $(L \pi)$ as the nucleus changes from initial state $i$ to final state $f$. We denote this transition amplitude as $T_{f i}(L \pi)$. Diagrams (b) and (c) portray the elastic interaction of the photon with each of the bound electrons; they differ only in the relative time ordering. The initial and final state " 0 " of the electron are identical, with the photon again emitted with multipolarity $(L \pi)$. The nuclear radiation induces currents in the atomic electrons, which then also radiate. The sum of the transition amplitudes for graphs (b) and (c) is denoted by $\delta T_{f i}(L \pi)$, and, as will be shown later, the total transition amplitude for the deexcitation can be written as

$$
\begin{aligned}
T_{f i}(L \pi)+\delta T_{f i}(L \pi) & =T_{f i}(L \pi)[1+\rho(L \pi)+i \xi(L \pi)] \\
& \approx T_{f i}(L \pi) e^{i \xi(L \pi)}
\end{aligned}
$$

with both $\rho$ and $\xi \ll 1$. This modifies the observed mixing ratio for the transition to

$$
\delta= \pm|\delta| e^{i(\eta+\xi)},
$$

with $\xi=\xi(L \pi)-\xi\left(L^{\prime} \pi^{\prime}\right)$, so that the relative phase measured in these experiments is actually $\eta+\xi$.

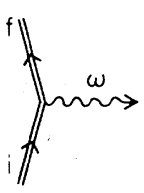

(a)

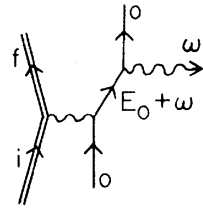

(b)

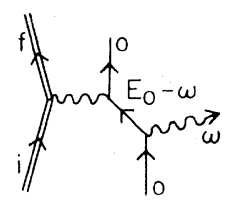

(c)
FIG. 1. Diagrammatic contributions to the amplitude for photon emission in a nuclear transition. The external wavy lines represent the emitted photon and the internal lines indicate virtual photon exchange. The single straight lines represent the electron, and the double lines the nucleus. 
Consequently, the knowledge of the interference parameter $\xi$ is essential for determining the true magnitude of the time-reversal violation, $\eta$.

Gimlett et al. ${ }^{6}$ have recently measured $\eta+\xi$ $=(-4.7 \pm 0.3) \times 10^{-3}$ for the $129.5 \mathrm{keV} E 2-M 1$ transition in ${ }^{191} \mathrm{Ir}$. Their result is to be compared with the theoretical value $\xi=-3.7 \times 10^{-3}$ calculated by Goldwire and Hannon, ${ }^{1}$ including an estimate of the $M$-shell contributions. This significant discrepancy between experiment and theory, or in other words an apparent evidence for time-reversal violation, was the primary motivation for the present work.

The parameter $\xi$ is also important for measurements of dispersion in Mössbauer absorption spectra. In transmission experiments, the total attenuation of the beam is due to the absorptive parts of scattering diagrams (a) through (d) in Fig. 2. Diagram (a) shows the direct nuclear resonant scattering, diagrams (b) and (c) represent the interaction of the radiation with atomic electrons either preceding or following the nuclear resonant scattering, and diagram (d) shows direct scattering of photons by the electrons. Using the optical theorem, one can verify that the interference between the nuclear resonant absorption and the interaction with the atomic electrons yields an attenuation cross section

$$
\sigma=\frac{\sigma_{0}(1-2 \xi x)}{1+x^{2}}+\sigma_{e},
$$

where $\sigma_{0}$ is the nuclear absorption cross section on resonance, and $x$ is the deviation of the photon energy from the resonance energy in units of the transition half-width. The quantity $\sigma_{e}$ is the slowly varying total cross section for photoelectric absorption and elastic plus inelastic scattering from atomic electrons.

Equation (4) shows that the interference parameter produces an asymmetry in the absorption line shape. Such an asymmetry was first observed by Sauer, Matthias, and Mössbauer, ${ }^{7}$ and has been treated theoretically by Trammel and Hannon ${ }^{2,3}$ and Kagan et al. ${ }^{8}$ It is also possible to measure

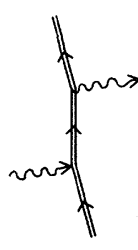

(a)

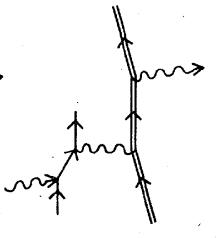

(b)

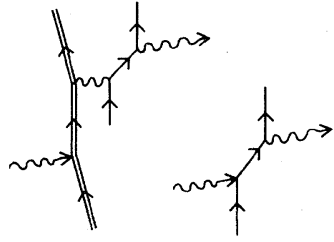

(d)
FIG. 2. Diagrams contributing to the scattering of a photon by a Mössbauer nucleus and its atomic electrons. contributions to the interference parameter by observing either ejected electrons (which reflect interference between photoelectrons and conversion electrons) or scattered $\gamma$ rays (which reflect interference between resonant scattering from the nucleus and nonresonant scattering from the electrons). In comparing calculated and measured values of $\xi$ for these experiments, it is necessary to properly take into account the effect of absorber thickness. This will be discussed in more detail below.

As pointed out earlier, the primary motivation of our present study was the apparent time-reversal violation observed by Gimlett et $a l .^{6} \mathrm{We}$ have rederived the formulas for the phase shift without approximations, particularly for the scattering part of $\xi$. Our numerical calculation is also more careful than that of Ref. 1 ; in particular, we use the more precise Hartree-Fock treatment of the bound and continuum electron wave functions. The more accurate handling of the electron wave functions enabled us to extend the calculations of $\xi$ to low energies, inaccessible in Ref. 1. It is with these low energy nuclear transitions that many of the precise Mössbauer measurements of $\xi$ are conducted. In addition, our exact treatment of the scattering part of $\xi$ is applicable at energies well above $200 \mathrm{keV}$, where atomic interference effects least obscure any possible time-reversal violation. In general, we find very good agreement with experiment, not only for the $129.5-\mathrm{keV}$ transition in ${ }^{191} \mathrm{Ir}$ where our calculated phase shift fully explains the observations, ${ }^{6}$ but also in numerous Mössbauer transitions.

In Sec. II we present theoretical expressions for the interference parameter $\xi$. A description of our calculation together with numerical results is given in Sec. III. We compare our results for $\xi$ with several time-reversal violation and Mössbauer absorption experiments in Sec. IV. The Appendix outlines the derivation of the formulas presented in Sec. III.

\section{EXPRESSIONS FOR THE INTERFERENCE PARAMETER}

We begin by considering the contribution to the amplitude for nuclear deexcitation by diagrams (a) through (c) in Fig. 1. The direct emission of a photon of wave vector $\vec{k}$, frequency $\omega=|\vec{k}|$, normalization $A$, and polarization $\vec{\epsilon}$ induced by the nuclear electromagnetic transition current $\vec{j}_{n}(\vec{x})$ is

$$
T_{f i}=2 \pi i \int d \overrightarrow{\mathrm{x}} \overrightarrow{\mathrm{j}}_{n}(\overrightarrow{\mathrm{x}}): A \vec{\epsilon} e^{-i \overrightarrow{\mathrm{k}} \cdot \overrightarrow{\mathrm{x}}},
$$

where $i$ and $f$ are the initial and final states of the 
nucleus, respectively, and $\omega=E_{i}-E_{f}$ is the energy of the transition.

We represent the sum of diagrams (b) and (c) as $\delta T_{f i}$, where in order for the processes to be indistinguishable from direct emission, the initial and final electron bound states are identical and the emitted photon is still described as above. Thus,

$$
\begin{aligned}
\delta T_{f i}=2 \pi i \int & \int \overrightarrow{\mathrm{x}} d \overrightarrow{\mathrm{y}} \frac{1}{4 \pi} \frac{e^{i \omega|\overrightarrow{\mathrm{y}}-\overrightarrow{\mathrm{x}}|}}{|\overrightarrow{\mathrm{y}}-\overrightarrow{\mathrm{x}}|} \\
& \times\left[\overrightarrow{\mathrm{j}}_{n}(\overrightarrow{\mathrm{x}}) \cdot \overrightarrow{\mathrm{j}}_{e}(\overrightarrow{\mathrm{y}})-\rho_{n}(\overrightarrow{\mathrm{x}}) \rho_{e}(\overrightarrow{\mathrm{y}})\right] .
\end{aligned}
$$

The electron current and density are defined as

$$
\begin{gathered}
\overrightarrow{\mathrm{j}}_{e}(\overrightarrow{\mathrm{y}}, \omega)=\sum_{0}\left[-e^{2} A \int d \overrightarrow{\mathrm{z}} \varphi_{0}^{*}(\overrightarrow{\mathrm{z}}) e^{-i \overrightarrow{\mathrm{k}} \cdot \overrightarrow{\mathrm{z}} \vec{\epsilon}} \cdot \vec{\alpha} G_{E_{0}{ }^{+\omega}}(\overrightarrow{\mathrm{z}}, \overrightarrow{\mathrm{y}})\right. \\
\times \vec{\alpha} \varphi_{0}(\overrightarrow{\mathrm{y}}) \\
-e^{2} A \int d \overrightarrow{\mathrm{z}} \varphi_{0}^{*}(\overrightarrow{\mathrm{y}}) \vec{\alpha} G_{E_{0}-\omega}(\overrightarrow{\mathrm{y}}, \overrightarrow{\mathrm{z}}) \\
\times e^{\left.-i \overrightarrow{\mathrm{k}} \cdot \overrightarrow{\mathrm{z}} \vec{\epsilon} \cdot \vec{\alpha} \varphi_{0}(\overrightarrow{\mathrm{z}})\right]}
\end{gathered}
$$

$$
\begin{gathered}
\rho_{e}(\overrightarrow{\mathrm{y}}, \omega)=\sum_{0}\left[-e^{2} A \int d \overrightarrow{\mathrm{z}} \varphi_{0}^{*}(\overrightarrow{\mathrm{z}}) e^{-i \overrightarrow{\mathrm{k}} \cdot \overrightarrow{\mathrm{z}} \vec{\epsilon} \cdot \vec{\alpha}} G_{E_{0}+\omega}(\overrightarrow{\mathrm{z}}, \overrightarrow{\mathrm{y}})\right. \\
\times \varphi_{0}(\overrightarrow{\mathrm{y}}) \\
-e^{2} A \int d \overrightarrow{\mathrm{z}} \varphi_{0}^{*}(\overrightarrow{\mathrm{y}}) G_{E_{0}-\omega}(\overrightarrow{\mathrm{y}}, \overrightarrow{\mathrm{z}}) \\
\times e^{\left.-i \overrightarrow{\mathrm{k}} \cdot \overrightarrow{\mathrm{z}} \overrightarrow{\mathrm{\epsilon}} \cdot \vec{\alpha} \varphi_{0}(\overrightarrow{\mathrm{z}})\right] .}
\end{gathered}
$$

Here the summation is over all bound electron wave functions $\varphi_{0}$ which satisfy the Dirac equation $[\vec{\alpha} \cdot \overrightarrow{\mathrm{p}}+\beta m+e V(\overrightarrow{\mathrm{r}})] \varphi_{0}(\overrightarrow{\mathrm{r}})=E_{0} \varphi_{0}(\overrightarrow{\mathrm{r}})$. The electron charge is $e, G_{E_{0^{ \pm}} \omega}$ is the electron Green's function of energy $E_{0} \pm \omega$ in the potential $V(\vec{r})$ of the nucleus and the remaining electrons, and $\rho_{n}(\overrightarrow{\mathrm{x}})$ is the nuclear electromagnetic transition density. Equation (6) expresses $\delta T_{f i}$ in a form very similar to that of internal conversion, but with $\vec{j}_{e}$ and $\rho_{e}$ having more complicated forms.

In the Appendix we outline the multipole decomposition of the photon plane wave and Green's function, and the construction of the electron propagators $G_{E_{0} \pm \omega}$. The total transition amplitude $T_{f i}+\delta T_{f i}$ can be written as a sum of amplitudes for the emission of photons of specific multipolarities:

$$
T_{f i}+\delta T_{f i}=\sum_{L, \pi}\left(T_{f i}+\delta T_{f i}\right)_{L, \pi},
$$

where for the magnetic case we obtain

$$
\left(T_{f i}+\delta T_{f i}\right)_{L, \text { mas }}=\sum_{M} 2 \pi i\left[\int d \overrightarrow{\mathrm{x}} \overrightarrow{\mathrm{j}}_{n}(\overrightarrow{\mathrm{x}}) \cdot \overrightarrow{\mathrm{a}}_{L M}^{(0)} *(\overrightarrow{\mathrm{x}})\right]\left\{A\left[\vec{\epsilon} \cdot \overrightarrow{\mathrm{Y}}_{L M}{ }^{(0)}(\hat{k})\right]+\frac{i \omega}{16 \pi^{2}} \int d \overrightarrow{\mathrm{y}} \overrightarrow{\mathrm{j}}_{e}(\overrightarrow{\mathrm{y}}) \cdot \overrightarrow{\mathrm{b}}_{L M}^{(0)}(\overrightarrow{\mathrm{y}})\right\},
$$

and for the electric case

$$
\begin{array}{rl}
\left(T_{f i}+\delta T_{f i}\right)_{L, \text { el ec }}=\sum_{M} & 2 \pi i\left[\int d \overrightarrow{\mathrm{x}} \overrightarrow{\mathrm{j}}_{n}(\overrightarrow{\mathrm{x}}) \cdot \overrightarrow{\mathrm{a}}_{L M}^{(1) *}(\overrightarrow{\mathrm{x}})\right] \\
\times\left(A\left[\vec{\epsilon} \cdot \overrightarrow{\mathrm{Y}}_{L M}^{(1)}(\hat{k})\right]+\frac{i \omega}{16 \pi^{2}} \int d \overrightarrow{\mathrm{y}}\left\{\overrightarrow{\mathrm{j}}_{e}(\overrightarrow{\mathrm{y}}) \cdot\left[\overrightarrow{\mathrm{b}}_{L M}^{(1)}(\overrightarrow{\mathrm{y}})+\left(\frac{L}{L+1}\right)^{1 / 2} \overrightarrow{\mathrm{b}}_{L M}^{(-1)}(\overrightarrow{\mathrm{y}})\right]\right.\right. \\
\left.\left.\quad-\rho_{e}(\overrightarrow{\mathrm{y}})\left(\frac{L}{L+1}\right)^{1 / 2} \varphi_{L M}(\overrightarrow{\mathrm{y}})\right\}\right) .
\end{array}
$$

Here the $\overrightarrow{\mathrm{Y}}_{L M}$ 's are the vector spherical harmonics, the $\overrightarrow{\mathrm{a}}_{L M}$ 's and $\overrightarrow{\mathrm{b}}_{L M}$ 's are the vector multipole fields, and the $\varphi_{L M}$ 's are the scalar multipole fields as defined in Akhiezer and Berestetskii. ${ }^{9}$ In $\mathrm{Eq}$. (A14) we show that the total transition amplitude $T_{f i}+\delta T_{f i}$ for each multipolarity $(L \pi)$ given in Eqs. (9) and (10) can be expressed as Eq. (2a). This defines $\xi$ and $\rho$.

As is clear from Fig. 1 and Eqs. (7), we have only considered processes contributing to order $e^{2}$ in the amplitude. Neglecting even higher order diagrams is justified by the smallness of the fine structure constant. The parameter $\rho$ is in general much less than one, and is of little significance because it occurs in the combination $1+\rho$.
In contrast, the interference parameter $\xi(L \pi)$, although small, plays an important role because of its imaginary character.

It has been shown previously ${ }^{1}$ that $\xi(L \pi)$ is a sum of two terms: the conversion phase $\xi_{c}$ and the scattering phase $\xi_{s}$. These imaginary parts of the transition amplitude arise from the singularities of the electron and photon Green's functions, respectively. We now discuss each of these contributions in greater detail.

$$
\text { A. } \xi_{c} \text {-the conversion phase }
$$

The electron propagator has a singularity when its energy equals that of a bound or free energy 
eigenstate. As indicated in Eqs. (7), we evaluate the Green's function $G$ for energies $E_{0}+\omega$ and $E_{0}-\omega$, where $E_{0}$ is the energy of each bound electron. For the transition energies which we consider here $(\omega<2 m)$, pair production is forbidden and only $E_{0}+\omega$ can coincide with the energy of a continuum eigenstate. This is by definition when $E_{0}+\omega$ is greater than the electron rest mass. Note that we neglect terms where $E_{0}+\omega$ coincides exactly with an unoccupied bound state. Thus, the only contributions to the conversion phase come from diagram (b) of Fig. 1. This is shown schematically in Fig. 3(a), suggesting that $\xi_{c}$ may be written as a product of two electron matrix elements: internal conversion followed by the inverse photoeffect. As is well known, the process of internal conversion is strongly energy dependent with those states just above threshold of greatest importance. We expect this same behavior for the conversion phase.

We define the solutions of the Dirac equation for the combined nuclear and atomic potential to be

$$
\varphi_{0}(\vec{y})=\frac{1}{y}\left(\begin{array}{l}
g_{\kappa_{0}}(y) \chi_{k_{0} \mu_{0}}(\hat{y}) \\
i f_{k_{0}}(y) \chi_{-k_{0} \mu_{0}}(\hat{y})
\end{array}\right),
$$

where $g_{k_{0}}$ and $f_{k_{0}}$ are the regular solutions to the radial Dirac equation:

$$
\begin{aligned}
& \frac{d g_{\kappa_{0}}}{d y}+\frac{\kappa_{0}}{y} g_{\kappa_{0}}=+\left[E_{0}+m-e V(y)\right] f_{\kappa_{0}}, \\
& \frac{d f_{\kappa_{0}}}{d y}-\frac{\kappa_{0}}{y} f_{\kappa_{0}}=-\left[E_{0}-m-e V(y)\right] g_{\kappa_{0}},
\end{aligned}
$$

and the $\chi$ 's are angular momentum spinors with total angular momentum $\left|\kappa_{0}\right|-\frac{1}{2}$ and the magnetic quantum number $\mu_{0}$.

For the magnetic multipoles, the conversion phase can be written as

$$
\xi_{c}(L, \pi=\operatorname{mag})=-\sum_{\kappa_{0} * k} A_{\kappa_{0^{k}}}(m) R_{\kappa_{0^{k}}}(m) S_{\kappa_{0^{k}}}(m),
$$

where the summation over $\kappa_{0}$ includes all fully occupied subshells (the role of valence electrons is not significant for this problem), and the summation over $\kappa$ represents all the final continuum

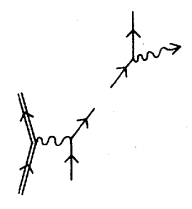

(a)

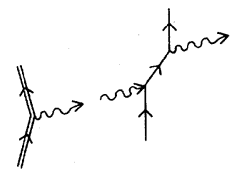

(b)

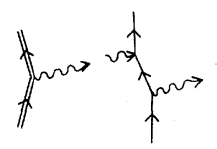

(c)
FIG. 3. Contributions to the interference parameter $\xi$. Diagram (a) represents the conversion contribution, and diagrams (b) and (c) the scattering portion of the phase.

states which are accessible to the initial state by the multipole selection rules. The other quantities appearing in $\mathrm{Eq}$. (13) are

$$
\begin{aligned}
A_{\kappa_{0} \kappa}(m)= & \pi \alpha \omega \frac{1}{L(L+1)}\left(\kappa+\kappa_{0}\right)^{2}(2 j+1)\left(2 j_{0}+1\right) \\
& \times\left(\begin{array}{ccc}
j_{0} & j & L \\
\frac{1}{2} & -\frac{1}{2} & 0
\end{array}\right)^{2}, \\
R_{\kappa_{0} k^{k}}(m) & =\int_{0}^{\infty} d r y_{L}(\omega r)\left[f_{k} g_{\kappa_{0}}+g_{k} f_{k_{0}}\right] \\
S_{\kappa_{0} k}(m) & =\int_{0}^{\infty} d r j_{L}(\omega r)\left[f_{k} g_{\kappa_{0}}+g_{k} f_{\kappa_{0}}\right]
\end{aligned}
$$

Here, $\alpha=e^{2} / 4 \pi$ is the fine structure constant, $j_{L}$ and $y_{L}$ are the regular and irregular spherical Bessel functions, $j_{0}$ and $j$ are the angular momenta for the initial and final electron states respectively, $f_{\kappa_{0}}$ and $g_{\kappa_{0}}$ are the bound state radial wave functions, and $f_{k}, g_{k}$ are the continuum wave functions which are regular at the origin. The normalization of the continuum states is defined in Eqs. (A9) of the Appendix. For comparison, the internal conversion coefficient is given by Rösel et al. ${ }^{10}$ as

$$
\alpha_{m}=\sum_{\kappa_{0}{ }^{* k}} A_{\kappa_{0} k}(m)\left[{R_{\kappa_{0} k}}^{2}(m)+S_{\kappa_{0} k}{ }^{2}(m)\right] .
$$

Similarly, for electric transitions one obtains

where

$$
\xi_{c}(L, \pi=\mathrm{elec})=-\sum_{\kappa_{0}, k} A_{\kappa_{0}{ }^{k}}(e) R_{\kappa_{0^{k}}}(e) S_{\kappa_{0} k}(e),
$$

$$
A_{\kappa_{0} k}(e)=\frac{1}{\left(\kappa+\kappa_{0}\right)^{2}} A_{\kappa_{0} \kappa}(m),
$$

$$
\begin{aligned}
& R_{\kappa_{0} \kappa}(e)=\int_{0}^{\infty} d r\left[-L y_{L}(\omega r)\left(g_{\kappa_{0}} g_{k}+f_{\kappa_{0}} f_{k}\right)-L y_{L-1}(\omega r)\left(f_{\kappa_{0}} g_{\kappa}-g_{\kappa_{0}} f_{k}\right)\right. \\
& \left.+\left(\kappa-\kappa_{0}\right) y_{L-1}(\omega r)\left(f_{\kappa_{0}} g_{\kappa}+g_{\kappa_{0}} f_{\kappa}\right)\right], \\
& S_{k_{0} k}(e)=\left(\frac{L+1}{2 L+1}\right) \int_{0}^{\infty} d r\left[-L j_{L+1}(\omega r)\left(f_{k_{0}} g_{k}-g_{\kappa_{0}} f_{k}\right)-L j_{L-1}(\omega r)\left(f_{\kappa_{0}} g_{k}-g_{\kappa_{0}} f_{k}\right)\right. \\
& -\frac{L}{L+1}\left(\kappa-\kappa_{0}\right) j_{L+1}(\omega r)\left(f_{\kappa_{0}} g_{k}+g_{\kappa_{0}} f_{k}\right) \\
& \left.+\left(\kappa-\kappa_{0}\right) j_{L-1}(\omega r)\left(f_{\kappa_{0}} g_{\kappa}+g_{\kappa_{0}} f_{\kappa}\right)\right] \text {. }
\end{aligned}
$$


The electric conversion coefficient is given by

$$
\alpha_{e}=\sum_{\kappa_{0}, k} A_{\kappa_{0} k}(e)\left[R_{\kappa_{0} k^{k}}{ }^{2}(e)+S_{\kappa_{0} k^{k}}{ }^{2}(e)\right] .
$$

In comparing our expression for the electric conversion phase with the formula of Hannon and Trammell, ${ }^{4}$ we have found an apparent error in their result. Their Eqs. (19) or (21) should contain an additional factor of $(2 L+2 / 2 L+1)$. The formula for the electric conversion phase in the more recent Ref. 1 can be shown to be equivalent to our expression.

\section{B. $\xi_{s}$-the scattering phase}

This contribution to the imaginary part of the transition amplitude for photon emission occurs when the photon Green's function is singular. This corresponds to the photon being real as follows from the expression for the propagator:

$$
\frac{\exp (i \omega|\overrightarrow{\mathrm{y}}-\overrightarrow{\mathrm{x}}|)}{|\overrightarrow{\mathrm{y}}-\overrightarrow{\mathrm{x}}|}=\frac{4 \pi}{(2 \pi)^{3}} \int d \overrightarrow{\mathrm{q}} \frac{\exp [i \overrightarrow{\mathrm{q}} \cdot(\overrightarrow{\mathrm{y}}-\overrightarrow{\mathrm{x}})]}{q^{2}-\omega^{2}-i \epsilon}
$$

Diagrams (b) and (c) of Fig. 3 show the emission of a real photon by the nucleus, which then scatters from all the bound atomic electrons. This scattering is elastic with the photon and electron states being unchanged by the interaction. The following expressions can be derived exactly using relativistic electron theory.

For the magnetic case we obtained

$$
\begin{aligned}
\xi_{s}(L, \pi & =\operatorname{mag}) \\
& =-\sum_{\kappa_{0}{ }^{*}} A_{\kappa_{0} \kappa}(m)\left[T_{\kappa_{0} \kappa}\left(E_{0}+\omega\right)-T_{\kappa_{0} \kappa}\left(E_{0}-\omega\right)\right],
\end{aligned}
$$

with

$$
\begin{aligned}
T_{\kappa_{0} \kappa}=\int_{0}^{\infty} & d z j_{L}(\omega z)\left(\bar{g}_{\kappa} f_{\kappa_{0}}+\bar{f}_{\kappa} g_{\kappa_{0}}\right)_{z} \\
& \times \int_{0}^{z} d y j_{L}(\omega y)\left(g_{\kappa} f_{\kappa_{0}}+f_{\kappa} g_{\kappa_{0}}\right)_{y} .
\end{aligned}
$$

As before, $\kappa_{0}$ labels the initial bound electron states, $\kappa$ labels the intermediate states of energies $E_{0}+\omega$ and $E_{0}-\omega$ which can be connected to the initial state via the selection rules, and the subscript $y$ or $z$ denotes the argument of the radial wave functions. The intermediate electron states can either be continuum wave functions with energy greater than the electron mass or quasibound wave functions with energy less than the electron mass. $A_{\kappa_{0}}(m)$ is the angular factor given in Eq. (14), $f_{\kappa_{0}}$ and $g_{\kappa_{0}}$ are again the bound state wave functions, $\bar{f}_{k}$ and $\bar{g}_{k}$ are the wave functions which are irregular at the origin, and $f_{k}$ and $g_{k}$ are regular at the origin.

The analogous expression for the electric scattering phase is rather lengthy and is presented elsewhere. ${ }^{11}$

Goldwire and Hannon ${ }^{1}$ have approximated the scattering phase as a sum of terms due to Thomson scattering (resulting from the $A^{2}$ interaction in the nonrelativistic reduction) and anomalous scattering (arising from the $\vec{j} \cdot \vec{A}$ interaction). They state that the Thomson term is dominant unless the transition is within a few $\mathrm{eV}$ of an absorption edge. We have found this to be true numerically even at higher energies where the approximation is no longer valid.

\section{NUMERICAL RESULTS}

In our calculations of the conversion and scattering phases, the potential $V(r)$ was taken to be the appropriate Dirac-Hartree-Slater potential for each atom. The potential also included a simple correction for finite nuclear size. A fifthorder predictor-corrector integration method was used to solve the coupled differential Eqs. (12). The bound state eigenfunctions were obtained by choosing $E_{0}$ so that two solutions matched at some intermediate radius: a regular one integrated outward from the origin and an exponentially decaying one, integrated inward from a very large radius. For the continuum and quasibound wave functions, we integrated the regular function outward from the origin, normalized at a distant point, and then integrated inward to obtain the irregular wave function.

In Ref. 1, the conversion phases were calculated for the $K$ and $L$ shells only. Unscreened, point nucleus, relativistic wave functions were used for the $K$ shell, while a table of screened internal conversion matrix elements was employed for the $L$ shell. The Thomson contribution to the scattering phase in Ref. 1 was calculated using published relativistic radial densities.

The major part of the conversion phase comes from those innermost bound states above conversion threshold. The outer shells also contribute, but in a rapidly decreasing manner. This behavior is demonstrated in Fig. 4 where the contributions of various shells to the conversion phase for two representative transitions are graphed. The variation of the scattering phase with shell is much slower with no threshold behavior. We used the exact relativistic expression for those states providing most (70-90\%) of the scattering phase. For the remaining states, we employed the much simpler Thomson approximation of Ref. 1. In 


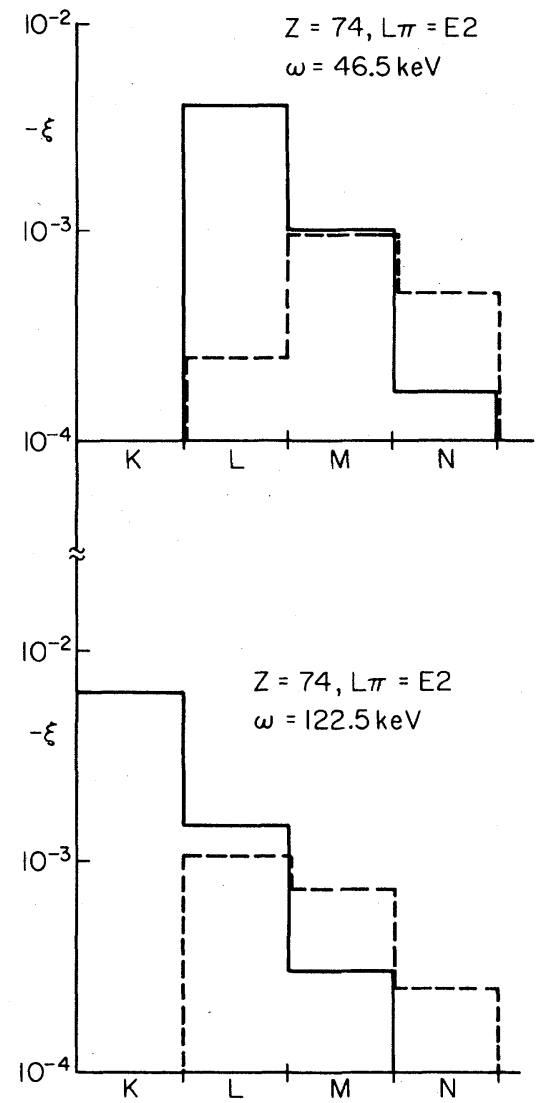

FIG. 4. Phase $\xi$ for two representative $E 2$ transitions in tungsten. Contributions of different electron shells are shown for the 46.5 and $122.5 \mathrm{keV}$ transitions in (a) and (b), respectively. Full histogram-conversion phase; dashed histogram-scattering phase.

Fig. 4 we display for the same transitions the contributions to the scattering phase for various atomic shells.

In general, our calculations agree with the conversion and scattering phases of Goldwire and Hannon. ${ }^{1}$ However, there are instances of disagreement, particularly for some $K$ shell conversion phases. These must be considered in the comparison with experiments in Sec. IV, particularly regarding the time-reversal violation test in ${ }^{191}$ Ir. ${ }^{6}$ Tables I-IV of Ref. 1 include nuclear transition energies up to $200 \mathrm{keV}$. Because both conversion and scattering phases decrease with increasing gamma energy, it may be desirable to perform time-revérsal experiments at higher energies. Therefore, we have calculated the phases at energies of 200,500, and $800 \mathrm{keV}$, and for nuclear charges 50, 70, and 90. Figures $5(\mathrm{a})-5$ (c) present these results for $E 1, M 1$, and E2 type transitions, respectively. These figures together with Tables I-IV of Ref. 1, should give adequate estimates of $\xi$ for any proposed experiments. However, in comparing theory with experiment, a more careful treatment of each transition is necessary. This is given in the next section for the existing experimental results.

There are several independent checks of our numerical work. These include comparisons with (a) experimental electron binding energies, (b) tabulated internal conversion coefficients, and $(c)$ experimental photoelectric cross sections.

(a) Binding energies. A comparison of bound state energy eigenvalues tests both the quality of these wave functions and that of the potential used to calculate the continuum and quasibound wave functions. The maximum difference we observed between the experimental and our calculated binding energies is of the order $1 \%$ for $K, L$, and $M$ shells. This comparison is quite satisfactory considering that higher-order effects such as virtual pair production are not included in the atomic potential.

(b) Internal conversion coefficients. There is some similarity between the expressions for conversion phase and internal conversion coefficients, as will be noted in comparing Eqs. (13) and (15) or Eqs. (16) and (18). We have found that $R_{k_{0} k}$ is in general greater than $S_{k_{0}}$ by two or three orders of magnitude in the region where the internal conversion coefficient and conversion phase are largest. Therefore, the internal conversion coefficient is effectively a measure of $R_{\kappa_{0}}$ in Eqs. (14) and (17). A comparison of our calculated coefficients with tabulated values strongly tests one factor in the conversion phase expression, and the results of this check are quite satisfactory. Table I compares the total $E 1$ internal conversion coefficients for the $K, L$, and $M$ shells of selected $97.4,25.6$, and $6.21 \mathrm{keV}$ transitions, respectively, with the tabulated values of Rösel et $a l .^{10}$

(c) Photoelectric cross sections. As was mentioned in the discussion of the conversion phase, the second matrix element $S_{\kappa_{0} \kappa}$ is effectively the amplitude for photoelectric absorption of photons of multipolarity $(L \pi)$. This can also be seen in Eqs. (A1) and (A2) of Ref. 1 for absorption cross sections. In the energy region of interest, the experimental photoelectric cross section is dominated by the $E 1$ contribution. Thus, by comparing our theoretical results for the photoelectric absorption cross section (calculated via the expressions of Ref. 1) with experimental data or extrapolated values, ${ }^{12}$ we are only able to check the accuracy of $S_{k_{0} k}$ for $E 1$ transitions. This comparison is presented for three selected $E 1$ transitions in Table I, showing our calculated values to lie within the quoted error bars. (We note that the E2 contribution to absorption for the $97.4 \mathrm{keV}$ 

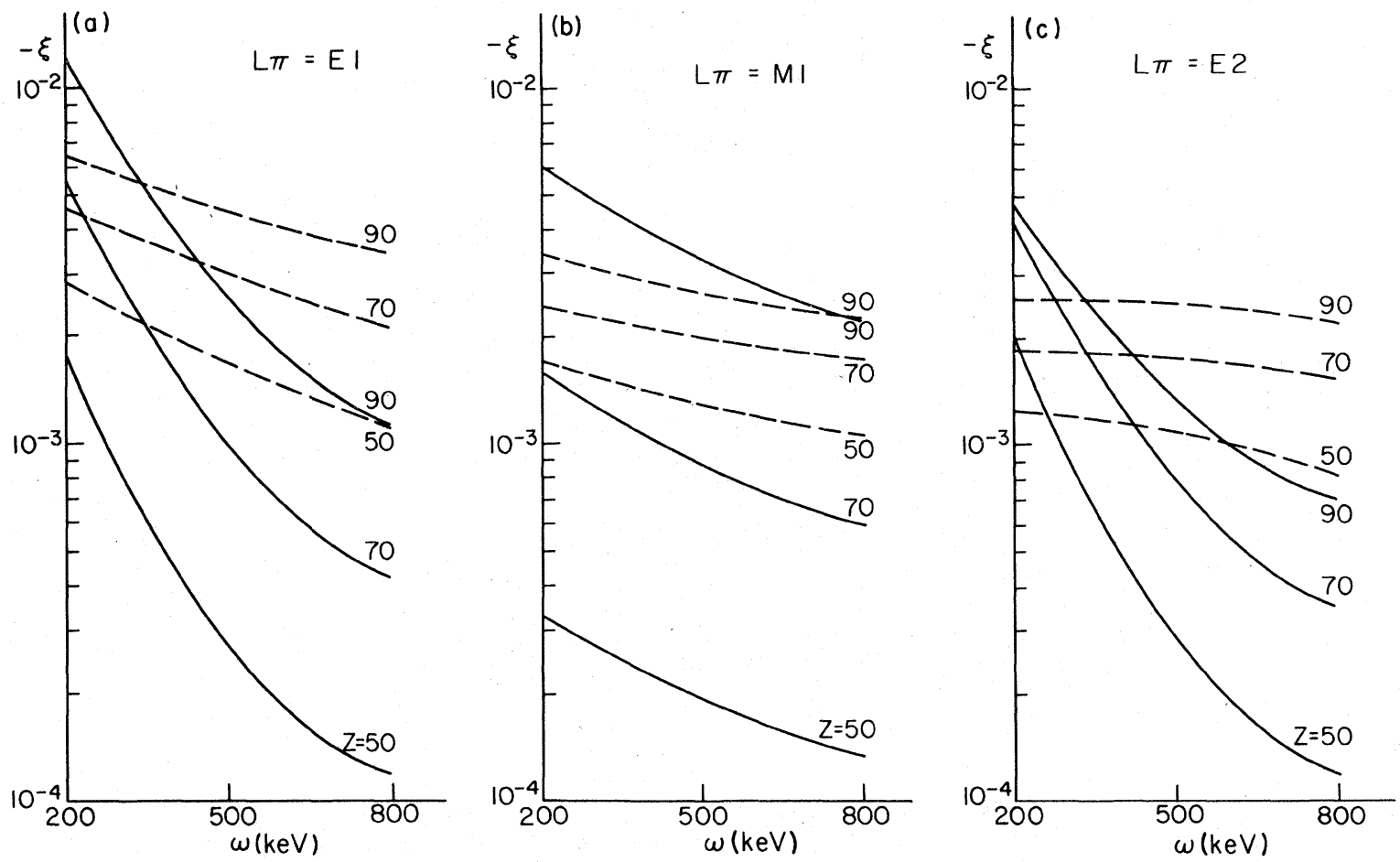

FIG. 5. Phase $\xi$ for transition energies between 200 and $800 \mathrm{keV}$ in nuclei of charge 50, 70, and 90. (a), (b), and (c) present results of calculation for $E 1, M 1$, and $E 2$ type transitions, respectively. Interference parameters for energies less than $200 \mathrm{keV}$ can be found in Ref. 1. Full curve-conversion phase; dashed curve-scattering phase.

transition, $1.04 \times 10^{2} \mathrm{~b} /$ atom, is included in our calculated value.)

We estimate the uncertainty in our values for the interference parameter $\xi(L \pi)$ to be of the order of $1-2 \%$. This takes into account, as best as possible, numerical aspects of the calculation and comparison with other relevant physical quantities. It must be noted, however, that due to subtraction there can be greater relative uncertainty in $\xi$ $=\xi(L \pi)-\xi\left(L^{\prime} \pi^{\prime}\right)$ measured in time-reversal violation experiments. The uncertainty in the scattering phase $\xi_{s}$ plotted in Fig. 5 is somewhat larger. Because Fig. 5 is meant mostly for orientation, we included only contributions of the $K, L$, and $M$ shells. This results in an underestimate of $\xi_{s}$ in Fig. 5 by about $10 \%$ at $200 \mathrm{keV}$, decreasing at higher energies.

\section{COMPARISON WITH EXPERIMENT}

In this section we compare our calculated values for the interference parameter with results of both time-reversal violation and Mössbauer absorption experiments. The computed phases $\xi$ include both conversion and scattering contributions as described in the preceding section. The only difference is that the scattering phase for some outer electron states was calculated according to the approximate expressions (16) and (17) of Ref. 1. These formulas require substantially less time for numerical computation and, as remarked earlier, generally give values close to those resulting from the exact expressions.

In Table II we present the experimentally measured values of $\xi$ together with their literature references. For Mössbauer experiments, it is necessary to correct the experimental results for absorber thickness. This effect, which causes the measured dispersion of absorption spectra to increase with absorber thickness, has been discussed by several authors; see, for example, Refs. 13, 14. An extrapolation to zero thickness is required for accurate comparison with our values. This correction can be significant, as demonstrated by the phase $\xi$ for the $6.21 \mathrm{keV} E 1$ transition of ${ }_{73}^{181} \mathrm{Ta}$ which was revised in Ref. 15 from the value $(-15.5 \pm 0.5) \times 10^{-2}$ given in Ref. 7 to $(-11 \pm 1) \times 10^{-2}$. A review of the literature has shown that most experimentalists have considered this effect and, when possible, corrected their values accordingly. A possible exception to this were Wagner et $a l .,{ }^{16}$ who alleged that their use 
TABLE I. Comparison of tabulated internal conversion coefficients and photoelectric absorption cross sections for selected $E 1$ transitions with results of the present calculation.

\begin{tabular}{ccclcl}
\hline \hline $\begin{array}{c}\text { Nuclear } \\
\text { charge }\end{array}$ & $\begin{array}{c}\text { Energy } \\
(\mathrm{keV})\end{array}$ & \multicolumn{1}{c}{$\alpha^{\mathrm{a}}$} & $\alpha_{\mathrm{TAB}^{\mathrm{b}}}$ & $\sigma(\mathrm{b} / \text { atom })^{\mathrm{c}}$ & $\sigma_{\mathbf{T} \text { AB }}(\mathrm{b} / \text { atom })^{\mathrm{d}}$ \\
\hline 63 & 97.4 & $\alpha_{K}=2.56(-1)$ & $2.56(-1)$ & $7.49(2)$ & $(7.57 \pm 0.38)(2)$ \\
66 & 25.6 & $\alpha_{L}=1.82(0)$ & $1.83(0)$ & $5.94(3)$ & $(6.36 \pm 0.64)(3)$ \\
73 & 6.21 & $\alpha_{M}=2.45(1)$ & $2.55(1)$ & $8.98(4)$ & $(9.31 \pm 0.93)(4)$ \\
\hline
\end{tabular}

\footnotetext{
${ }^{\mathrm{a}}$ Calculated total conversion coefficients for first contributing shell; $2.56(-1)$ denotes 2.56 $\times 10^{-1}$.

${ }^{b}$ Tabulated values from Ref. 10.

${ }^{\mathrm{C}}$ Calculated cross section for photoelectric absorption.

${ }^{\mathrm{d}}$ Tabulated values with uncertainties from Ref. 12.
}

of absorbers of thickness $t \leqslant 2$ required no correction (where $t$ is a dimensionless measure of thickness as defined, for example, in Ref. 14). However, finite thickness corrections to measurements performed at $t=2$ can be as large as $50 \% .^{14}$

For transitions of mixed E2-M1 multipolarity, the relevant phase measured in time-reversal experiments is $\xi=\xi(E 2)-\xi(M 1)$, and the phase determined by Mössbauer experiments is $\xi=[\xi(M 1)$ $\left.+\delta^{2} \xi(E 2)\right] /\left(1+\delta^{2}\right)$. We have used the mixing ratios $\delta$ given in the experimental references.

The agreement between calculated and measured values $^{6.14-24}$ is quite good, as portrayed graphically in Fig. 6. The largest discrepancies occur in the $46.5,100.1$, and $122.5 \mathrm{keV}$ transitions of tungsten measured by Wagner et al. ${ }^{16}$ Their failure to correct for nonzero thickness possibly explains this larger experimental values for the 100.1 and $122.5 \mathrm{keV}$ transitions. One would also have expected that the measured phases for the 99.1 and $100.1 \mathrm{keV} E 2$ transitions in tungsten to be nearly equal, as there are no threshold effects at this energy to cause such a difference. In both the $25.6 \mathrm{keV}$ transition of ${ }^{161} \mathrm{Dy}$ and the $97.4 \mathrm{keV}$ transition of ${ }^{153} \mathrm{Eu}$ we are in satisfactory agreement with one experimental result while differing significantly from the other. We include both measurements of each transition in Table II and Fig. 6. For the majority of these transitions, our values are quite close to those of Ref. 1. Those authors, however, were unable to calculate the interference parameter for the lowest energy transition-the $6.21 \mathrm{keV}$ line in tantalum. Our value for this Mössbauer transition is in excellent accord with experiment.

We have also computed phases arising from final state atomic effects in two recent time-reversal violation experiments. There is agreement with the $122.1 \mathrm{keV}$ mixed $E 2-M 1$ transition in ${ }_{26}^{57} \mathrm{Fe},{ }^{24}$ and the experimental value for the $364.5 \mathrm{keV}$ transition in xenon will be published in the near future, ${ }^{25}$ indicating no violation of time reversal at the level of the experimental uncertainty. We note that the near equality of $E 2$ and $M 1$ phases for the xenon transition causes the theoretical uncertainty $\left(0.01 \times 10^{-2}\right)$ to be the same magnitude as the expected phase difference.

Of greatest significance is our agreement with the observed phase shift for the $129.5 \mathrm{keV}$ transition of ${ }_{77}^{191} \mathrm{Ir}$. The calculated value of $(-4.3 \pm 0.4)$ $\times 10^{-3}$ (the indicated limits reflect the $2 \%$ uncertainty in both $E 2$ and $M 1$ phases) shows no discrepancy with the experimental value of $(-4.7 \pm 0.3)$ $\times 10^{-3}$. This removes the apparent evidence for time-reversal violation, which originated in a comparison with the values presented in Ref. 1 . We differ with Goldwire and Hannon ${ }^{1}$ primarily in the $K$-shell conversion phase for both multipolarities. Good agreement with binding energies and tabulated internal conversion coefficients for the $K$ shell verifies the accuracy of our calculations.

\section{ACKNOWLEDGMENTS}

We acknowledge helpful conversations with F. Boehm and J. Gimlett, and thank G. Wortman for alerting us to the effect of absorber thickness on Mössbauer absorption experiments. This work was supported in part by the National Science Foundation (PH77-21602 and PHY79-23638) and the U. S. Department of Energy (DE AC-03-76ER0063). S. E. Koonin was supported in part by the Alfred P. Sloan Foundation.

\section{APPENDIX: DERIVATION OF EXPRESSIONS FOR INTERFERENCE PARAMETER $\xi$}

This appendix outlines the steps taken to obtain the formulas for conversion and scattering phases in Sec. II. The method is in many respects similar to that used by $\operatorname{Rose}^{26}$ in discussing the process of internal conversion. One initially performs a multipole decomposition of the photon plane wave and photon Green's function which 
TABLE II. Comparison of experimental interfer ence parameters $\xi$ with results of the present calculation.

\begin{tabular}{|c|c|c|c|c|c|c|}
\hline Isotope & $\begin{array}{c}\omega \\
(\mathrm{keV})\end{array}$ & Multipolarity & $100 \xi^{\mathrm{a}}$ & \multicolumn{2}{|c|}{$100 \xi_{\mathrm{EXPT}}{ }^{\mathrm{b}}$} & Ref. \\
\hline${ }_{26}^{57} \mathrm{Fe}$ & 122.1 & $M 1+1.4 \% E 2$ & $\xi=-0.06$ & $\xi=-0.03$ & \pm 0.07 & 24 \\
\hline${ }_{32}^{73} \mathrm{Ge}$ & 13.3 & $E 2$ & -3.24 & -4.7 & \pm 1.0 & 20 \\
\hline \multirow[t]{2}{*}{${ }_{44}^{99} \mathrm{Ru}$} & 90.0 & $E 2+37 \% M 1$ & -0.60 & -0.33 & \pm 0.32 & 16 \\
\hline & & & $\xi=-0.57^{c}$ & $\xi=-0.43$ & \pm 0.50 & 21 \\
\hline${ }_{54}^{131} \mathrm{Xe}$ & 364.5 & $E 2+4.6 \% M 1$ & $\xi=-0.01^{c}$ & & $\mathrm{~d}$ & \\
\hline \multirow{2}{*}{${ }_{63}^{153} \mathrm{Eu}$} & 97.4 & $E 1$ & -2.03 & -1.1 & \pm 0.3 & 18 \\
\hline & & & & -1.4 & \pm 0.3 & 14 \\
\hline \multirow{2}{*}{${ }_{64}^{155} \mathrm{Gd}$} & 86.5 & $E 1$ & -2.48 & -2.5 & \pm 0.5 & 18 \\
\hline & 105.3 & $E 1$ & -1.85 & -1.8 & \pm 0.5 & 18 \\
\hline \multirow{3}{*}{${ }_{66}^{161} \mathrm{Dy}$} & 25.6 & $E 1$ & -3.97 & -3.5 & \pm 0.5 & 18 \\
\hline & & & & -3.2 & \pm 0.3 & 14 \\
\hline & 74.5 & $E 1$ & -3.40 & -3.0 & \pm 0.5 & 18 \\
\hline${ }_{68}^{166} \mathrm{Er}$ & 80.6 & $E 2$ & -1.37 & -1.60 & \pm 0.19 & 16 \\
\hline${ }_{70}^{170} \mathrm{Yb}$ & 84.3 & $E 2$ & -1.33 & -1.70 & \pm 0.38 & 16 \\
\hline${ }_{70}^{171} \mathrm{Yb}$ & 66.7 & $M 1+49 \% E 2$ & -0.75 & -1.00 & \pm 0.14 & 16 \\
\hline${ }_{72}^{180} \mathrm{Hf}$ & 93.3 & $E 2$ & -1.27 & -1.82 & \pm 0.48 & 16 \\
\hline${ }_{73}^{181} \mathrm{Ta}$ & 6.21 & $E 1$ & -12.3 & -11 & \pm 1 & 15 \\
\hline${ }_{74}^{182} \mathrm{~W}$ & 100.1 & $E 2$ & -1.22 & -1.71 & \pm 0.14 & 16 \\
\hline \multirow{2}{*}{${ }_{74}^{183} \mathrm{~W}$} & 99.1 & $E 2$ & -1.22 & -1.25 & \pm 0.17 & 16 \\
\hline & 46.5 & $M 1+0.6 \% E 2$ & -0.28 & -0.05 & \pm 0.06 & 16 \\
\hline${ }_{74}^{184} \mathrm{~W}$ & 111.1 & $E 2$ & -1.17 & -1.53 & \pm 0.29 & 16 \\
\hline${ }_{74}^{186} \mathrm{~W}$ & 122.5 & $E 2$ & -1.10 & -2.09 & \pm 0.36 & 16 \\
\hline${ }_{76}^{186} \mathrm{Os}$ & 137.2 & $E 2$ & -1.02 & -1.02 & \pm 0.25 & 16 \\
\hline${ }_{76}^{188} \mathrm{Os}$ & 155.0 & $E 2$ & -0.93 & -1.51 & \pm 0.49 & 16 \\
\hline \multirow[t]{2}{*}{${ }_{177}^{191} \mathrm{Ir}$} & 129.5 & $M 1+14 \% E 2$ & -0.69 & -0.50 & \pm 0.12 & 16 \\
\hline & & & $\xi=-0.43^{c}$ & $\xi=-0.47$ & \pm 0.03 & 6 \\
\hline${ }_{77}^{193} \mathrm{Ir}$ & 73.1 & $M 1+31 \% E 2$ & $\xi=-0.12^{c}$ & $\xi=0.11$ & \pm 0.38 & 22 \\
\hline${ }_{78}^{195} \mathrm{Pt}$ & 98.7 & $M 1$ & -0.70 & -1.1 & \pm 0.3 & 23 \\
\hline${ }_{79}^{197} \mathrm{Au}$ & 77.3 & $M 1+12.1 \% E 2$ & -0.40 & -0.414 & \pm 0.017 & 19 \\
\hline${ }_{92}^{236} \mathrm{U}$ & 45.3 & $E 2$ & +0.31 & +0.25 & \pm 0.75 & 16 \\
\hline${ }_{93}^{237} \mathrm{~Np}$ & 59.6 & $E 1$ & -3.18 & -3.4 & \pm 0.2 & 17 \\
\hline
\end{tabular}

${ }^{a}$ Calculated interference parameters $\xi$. Both conversion and scattering contributions included.

${ }^{\mathrm{b}}$ Measured values of $\xi$, together with experimental references.

${ }^{c}$ For time-reversal violation experiments, $\xi=\xi(E 2)-\xi(M 1)$.

d Experimental value to be published in the near future (Ref. 25).

occur in Eqs. (5) and (6), respectively:

$\vec{\epsilon} e^{-i \overrightarrow{\mathrm{k}} \cdot \overrightarrow{\mathrm{x}}}=\sum_{L, M}\left[\overrightarrow{\boldsymbol{\epsilon}} \cdot \overrightarrow{\mathrm{Y}}_{L M}^{(0)}(\hat{k}) \overrightarrow{\mathrm{a}}_{L M}^{(0) *}(\overrightarrow{\mathrm{x}})+\overrightarrow{\boldsymbol{\epsilon}} \cdot \overrightarrow{\mathrm{Y}}_{L M}^{(1)}(\hat{k}) \overrightarrow{\mathrm{a}}_{L M}^{(1) *}(\overrightarrow{\mathrm{x}})\right]$

$$
\begin{aligned}
\frac{e^{i \omega|\vec{y}-\vec{x}|}}{|\vec{y}-\vec{x}|} \vec{j}_{e}(\vec{y}) \cdot \vec{j}_{n}(\vec{x})=\frac{i \omega}{4 \pi} \sum_{L, M, t=0,1,-1} & {\left[\vec{j}_{n}(\vec{x}) \cdot \vec{a}_{L M}^{(t) *}(\vec{x})\right] } \\
\times & \times\left[\vec{j}_{e}(\vec{y}) \cdot \vec{b}_{L M}^{(t)}(\vec{y})\right],
\end{aligned}
$$




$$
\begin{aligned}
\frac{e^{i \omega|\vec{y}-\vec{x}|}}{|\vec{y}-\vec{x}|} \rho_{e}(\vec{y}) \rho_{n}(\vec{x})=4 \pi i \omega \sum_{L, M} & {\left[\rho_{n}(\vec{x}) j_{L}(\omega x) Y_{L M}^{*}(\hat{x})\right] } \\
& \times\left[\rho_{e}(\vec{y}) h_{L}(\omega y) Y_{L M}(\hat{y})\right] .
\end{aligned}
$$

The expansions in Eqs. (A2) and (A3) are valid for $x<y$, where $x$ and $y$ are the magnitudes of position vectors $\vec{x}$ and $\vec{y}$, respectively. The $\overrightarrow{\mathrm{Y}}_{L M}^{(0)}$ and $\overrightarrow{\mathrm{Y}}_{L M}^{(1)}$ are vector spherical harmonics of magnetic and electric type, respectively, the $\overrightarrow{\mathrm{a}}_{L M}^{(t)}$ 's and $\vec{b}_{L M}^{(t)}$ 's are the vector multipole fields of type magnetic, electric, and longitudinal for $t=0,1$,
-1 , respectively, as defined in Akhiezer and Berestetskii, ${ }^{9}$ and the $Y_{L M}$ 's are scalar spherical harmonics. These expressions lead immediately to the results for $T_{f i}+\delta T_{f i}$ given in Eqs. (9) and (10).

The other important element of the derivation involves construction of the Dirac propagator for the electron, which satisfies

$$
[E-\vec{\alpha} \cdot \overrightarrow{\mathrm{p}}-\beta m-e V(\overrightarrow{\mathrm{x}})] G_{E}(\overrightarrow{\mathrm{x}}, \overrightarrow{\mathrm{y}})=I \delta(\overrightarrow{\mathrm{x}}-\overrightarrow{\mathrm{y}}),
$$

where $I$ is the $4 \times 4$ unit matrix. We write $G_{E}(\vec{x}, \vec{y})$ in the following form:

$$
G_{E}(\mathrm{x}, \mathrm{y})=\sum_{\kappa, \mu} \frac{1}{x y}\left[\begin{array}{cc}
\chi_{\kappa \mu}(\hat{x}) \chi_{\kappa \mu}^{+}(\hat{y}) G_{++}(x, y) & -i \chi_{\kappa \mu}(\hat{x}) \chi_{-\kappa \mu}^{+}(\hat{y}) G_{+-}(x, y) \\
i \chi_{-\kappa \mu}(\hat{x}) \chi_{\kappa \mu}^{+}(\hat{y}) G_{-+}(x, y) & \chi_{-\kappa \mu}(\hat{x}) \chi_{-\kappa \mu}^{+}(\hat{y}) G_{---}(x, y)
\end{array}\right],
$$

as is possible for a spherically-symmetric potential $V(x)$. The radial Green's functions $G_{++}, G_{+- \text {, }}$ $G_{-+}, G_{--}$depend on $E$ and $\kappa$ (but not $\mu$ ), and satisfy the following coupled differential equations:

$$
\begin{aligned}
& -\left[E-m-e V(x) G_{++}\right]-\left(G_{-+}^{\prime}-\frac{\kappa}{x} G_{-+}\right)=\delta(x-y), \\
& G_{++}^{\prime}+\frac{\kappa}{x} G_{++}-[E+m-e V(x)] G_{-+}=0, \\
& -[E-m-e V(x)] G_{+-}-\left(G_{--}^{\prime}-\frac{\kappa}{x} G_{--}\right)=0, \\
& G_{+-}^{\prime}+\frac{\kappa}{x} G_{+-}-[E+m-e V(x)] G_{--}=\delta(x-y) .
\end{aligned}
$$

Here the primes denote derivatives with respect to

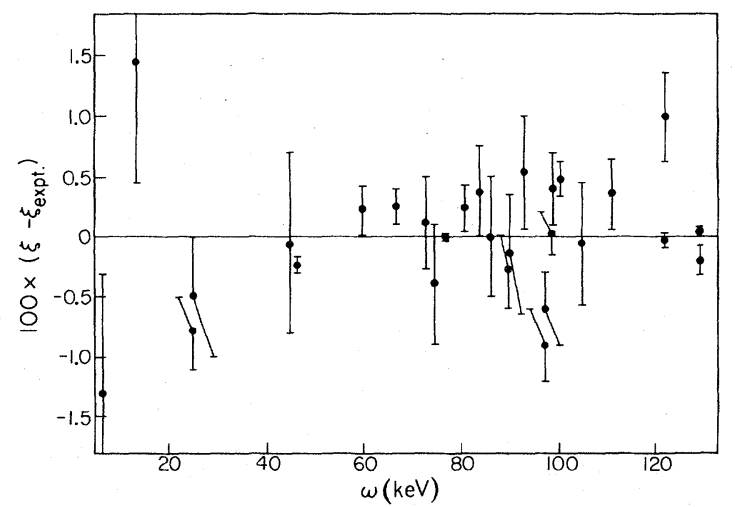

FIG. 6. Comparison of calculation with experiment for the time-reversal violation and Mössbauer absorption measurements listed in Table II. The energy of transition is plotted horizontally; the difference between the calculated and experimentally measured phase shifts $\left(\xi-\xi_{\text {EXPT }}\right)$, in units of $10^{-2}$, is plotted vertically. The error bars include only those of the experiment. the variable $x$. For the energies relevant to our calculations $(E>-m)$ these equations are solved by

$$
\left[\begin{array}{c}
G_{++}(x, y) \\
G_{-+}(x, y) \\
G_{+-}(x, y) \\
G_{--}(x, y)
\end{array}\right]=\frac{1}{W}\left[\begin{array}{cc}
g_{0}(x) g_{+}(y) & g_{+}(x) g_{0}(y) \\
f_{0}(x) g_{+}(y) & f_{+}(x) g_{0}(y) \\
g_{0}(x) f_{+}(y) & g_{+}(x) f_{0}(y) \\
f_{0}(x) f_{+}(y) & f_{+}(x) f_{0}(y) \\
x<y & x>y
\end{array}\right] .
$$

In the continuum case $(E>m)$

$$
p=\left(E^{2}-m^{2}\right)^{1 / 2}, \quad W=i p /(E+m)
$$

and the "large" radial wave functions $g_{0}$ (regular), $g$ - (irregular), and $g_{+}$(outgoing) solve equations

(12) with the asymptotic forms as $y \rightarrow \infty$ :

$$
\begin{aligned}
& g_{0} \sim\left(\frac{p(E+m)}{\pi}\right)^{1 / 2} y\left[\cos \delta^{\prime} j_{l}(p y)-\sin \delta^{\prime} y_{l}(p y)\right], \\
& g_{-} \sim\left(\frac{p(E+m)}{\pi}\right)^{1 / 2} y\left[\sin \delta^{\prime} j_{l}(p y)+\cos \delta^{\prime} y_{l}(p y)\right],
\end{aligned}
$$

$$
g_{+}=g_{0}+i g
$$

The orbital angular momentum $l$ is given by

$$
l=\left\{\begin{array}{l}
\kappa \text { for } \kappa>0 \\
-\kappa-1 \text { for } \kappa<0 .
\end{array}\right.
$$

The parameter $\delta^{\prime}$ is related to the conventional phase shift $\delta$ by $\delta^{\prime}=\delta+\pi(l+1) / 2$. This latter phase is defined by the asymptotic form for the regular wave function $g_{0}$ :

$$
g_{0} \sim\left(\frac{E+m}{\pi p}\right)^{1 / 2} \cos (p y+\delta)
$$

The asymptotic forms for the "small" radial func- 
tions $f_{0}, f_{-}, f_{+}$are obtained from $g_{0}, g_{-}, g_{+}$by the coupled differential equations (12) with the potential $V$ set equal to zero. It is usual in atomic calculations to employ the asymptotic forms of the radial wave functions represented by $\mathrm{Eq}$. (A11). However, this assumes that both the potential and centrifugal barrier are very small at the radius of normalization. In our case the centrifugal barrier was not in general negligible, and thus, we used these more appropriate asymptotic expressions (A9).

For the quasibound case $(-m<E<m)$ :

$$
p=\left(m^{2}-E^{2}\right)^{1 / 2}, \quad W=-p /(E+m)
$$

and the radial wave functions $g_{0}$ (regular) and $g_{+}$ (irregular) have the asymptotic forms

$g_{0} \sim \frac{e^{p y}}{2}\left[1-\frac{\kappa(\kappa+1)}{2} \frac{1}{p y}+O\left(\left(\frac{1}{p y}\right)^{2}\right)\right]$,
$g_{+} \sim\left(\frac{E+m}{\pi p}\right)^{1 / 2} e^{-p y}\left[1+\frac{\kappa(\kappa+1)}{2} \frac{1}{p y}+O\left(\left(\frac{1}{p y}\right)^{2}\right)\right]$.

The small functions $f_{0}$ and $f_{+}$are obtained from $g_{0}$ and $g_{+}$as described above.

When the solutions for $G_{E}(\overrightarrow{\mathrm{x}}, \overrightarrow{\mathrm{y}})$ given by Eqs. (A5) and (A7) are inserted into Eqs. (9) and (10), with the angular parts coupled correctly to those coming from the photon, the resulting expression for the magnetic case is

$$
\begin{aligned}
&\left(T_{f i}+\delta T_{f i}\right)_{L, \text { mag }} \\
&=\sum_{M} 2 \pi i\left(\int d \overrightarrow{\mathrm{x}} \overrightarrow{\mathrm{j}}_{n}(\overrightarrow{\mathrm{x}}) \cdot \overrightarrow{\mathrm{a}}_{L M}^{(0) *}(\overrightarrow{\mathrm{x}})\right) \cdot A\left[\vec{\epsilon} \cdot \overrightarrow{\mathrm{Y}}_{L M}^{(0)}(\hat{k})\right] \\
& \times\left(1-i \sum_{\kappa_{0}, \kappa} A_{\kappa_{0} k}(m) \int_{0}^{\infty} d z \int_{0}^{\infty} d y h_{L}(\omega y) j_{L}(\omega z)\right. \\
& \times\left(\left\{f_{\kappa_{0}}(z)\left[f_{\kappa_{0}}(y) G_{++}^{+}(z, y)+g_{\kappa_{0}}(y) G_{+-}^{+}(z, y)\right]+g_{\kappa_{0}}(z)\left[f_{\kappa_{0}}(y) G_{++}^{+}(z, y)+g_{\kappa_{0}}(y) G_{--}^{+}(z, y)\right]\right\}\right. \\
&\left.\left.+\left\{f_{\kappa_{0}}(z)\left[f_{\kappa_{0}}(y) G_{++}^{-}(y, z)+g_{\kappa_{0}}(y) G_{-+}^{-}(y, z)\right]+g_{\kappa_{0}}(z)\left[f_{\kappa_{0}}(y) G_{+-}^{-}(y, z)+g_{\kappa_{0}}(y) G_{--}^{-}(y, z)\right]\right\}\right)\right) .
\end{aligned}
$$

The superscripts + or - on the G's refer to energies $E_{0}+\omega$ or $E_{0}-\omega$, respectively. The imaginary part of the above expression gives the magnetic interference parameter $\xi(L \pi)$ as the sum of two parts: the conversion phase of Eq. (13) and the scattering phase of Eq. (20).
A similar procedure applies for the electric interference parameter; however, space does not permit the reproduction here of the lengthy scattering formula [analog of Eq. (20)]. The reader is referred to Ref. 11 for further details.
${ }^{1} \mathrm{H}$. C. Goldwire, Jr. and J. P. Hannon, Phys. Rev. B 16 , 1875 (1977).

${ }^{2}$ J. P. Hannon and G. T. Trammell, Phys. Rev. Lett. 21, 726 (1968).

${ }^{3}$ G. T. Trammell and J. P. Hannon, Phys. Rev. 180,337 (1969).

${ }^{4}$ J. P. Hannon and G. T. Trammell, Phys. Rev. $\underline{186}, 306$ (1969).

${ }^{5}$ S. P. Lloyd, Phys. Rev. 81,161 (1951).

${ }^{6}$ J. L. Gimlett, H. E. Henrikson, N. K. Cheung, and F. Boehm, Phys. Rev. Lett. 42, 354 (1979).

${ }^{7}$ C. Sauer, E. Matthias, and R. L. Mössbauer, Phys. Rev. Lett. 21, 961 (1968).

${ }^{8}$ Yu. Kagan, A. M. Afanas'ev, and V. K. Voitovetskii, Zh. Eksp. Teor. Fiz. Pis 'ma Red. 9 , 155 (1969) [JETP Lett. 9,91 (1969)].

${ }^{9}$ A. I. A $\bar{k} h i e z e r$ and V. B. Berestetskii, Quantum Electrodynamics (Wiley-Interscience, New York, 1965).

${ }^{10}$ F. Rösel, H. M. Fries, K. Alder, and H. C. Pauli, At. Data Nucl. Data Tables 21, 91 (1978).

${ }^{11}$ B. R. Davis, Ph.D. thesis, California Institute of Tech- nology, 1980 (unpublished).

${ }^{12}$ W. J. Veigele, At. Data 5, 51 (1973).

${ }^{13} \mathrm{~B}$. T. Cleveland and J. Heberle, Phys. Lett. $40 \mathrm{~A}, 13$ (1972).

${ }^{14} \mathrm{P}$. West, Nuc1. Instrum. Methods 101, 243 (1972).

${ }^{15}$ D. Salomon, P. J. West, and G. Weyer, Hyperfine Interactions $\underline{5}, 61$ (1977).

${ }^{16}$ F. E. Wagner, B. D. Dunlap, G. M. Kalvius, H. Schaller, R. Felscher, and H. Spieler, Phys. Rev. Lett. 28 530 (1972).

${ }^{17}$ L. Asch, W. Potzel, G. M. Kalvius, J. C. Spirlet, and W. Müller, Hyperfine Interactions 5 , 457 (1978).

${ }^{18}$ W. Henning, G. Bähre, and P. Kienle, Phys. Lett. B 31,203 (1970).

${ }^{19}$ D. J. Erickson, J. P. Prince, and L. D. Roberts, Phys. Rev. C 8, 1916 (1973).

${ }^{20}$ L. Pfeiffer, Phys. Rev. Lett. 38, 862 (1967).

${ }^{21}$ O. C. Kistner, Phys. Rev. Lett. 19, 872 (1967).

${ }^{22}$ M. Atac, B. Chrisman, P. Debrunner, and H. Frauenfelder, Phys. Rev. Lett. 20, 691 (1968).

${ }^{23}$ W. Potzel, F. E. Wagner, G. M. Kalvius, L. Asch, 
J. C. Spirlet, and W. Müller, in Proceedings of the Workshop on New Directions in Mössbauer Spectroscopy, Argonne, 1977, edited by Bilbert J. Perlow (AIP, New York, 1977).

${ }^{24}$ N. K. Cheung, H. E. Henrikson, and F. Boehm, Phys.
Rev. C 16, 2381 (1977).

${ }^{25} \mathrm{~J}$. L. Gimlett, private communication.

${ }^{26}$ M. E. Rose, Multipole Fields (Wiley, New York, 1955). 